\title{
THE BALMER LINES IN THE SEYFERT GALAXIES NGC 5548 AND NGC 4151
}

\author{
R. WEYMANN and R. CROMWELL \\ Steward Observatory, Tucson, Ariz., U.S.A.
}

\begin{abstract}
The profiles of the Balmer lines in NGC 5548 as reported by Dibai et al. (1968) were somewhat asymmetric, whereas those reported by Anderson (1970) are smooth and symmetric. We present profiles which are strongly asymmetric, resembling those of Dibai et al. Evidently electron scattering is not the sole principal broadening agent and we must deal with velocities $\sim 2500 \mathrm{~km} \mathrm{~s}^{-1}$ in a very small volume.

The transient nature of the P-Cygni type profiles in the Balmer lines of NGC 4151 has previously been noted (Cromwell and Weymann, 1970). These lines have since disappeared, at the resolution available to us, in a time of 3 months. A model in which frequent outbursts of shells or filaments produce transient features in the Balmer lines, while the accumulated material from past outbursts produces the relatively stable He I $\lambda 3889$ feature, seems the most plausible.
\end{abstract}

This contribution deals with two items: the marked asymmetry observed in the $\mathrm{H} \beta$ profile of NGC 5548, and the appearance and subsequent disappearance of displaced absorption cores in the Balmer lines of NGC 4151.

There have been two recent attempts to interpret the wide wings characteristic of the Balmer line profiles in Seyfert galaxies in terms of electron scattering occurring in very dense, very small regions of the nuclei of these objects (Weymann, 1970; Mathis, 1970).

According to profiles recently obtained by Anderson (1970), the $\mathrm{H} \beta$ profile in NGC 5548 is quite symmetrical and smooth, and can be matched quite well by the theoretical line profiles. This success in matching the profile thus supported the reality of the electron-scattering model.

During the interval December 1969-June 1970 however, we obtained several spectrograms of NGC 5548, all of which show a rather marked asymmetry. A representative tracing from one of these spectrograms is shown in Figure 1. Also included is the profile derived by Anderson, as well as a tracing of a spectrogram taken in May 1966 by Dibai et al. (1968). The same asymmetry is evident in this tracing, (though not as clearly as on our spectrograms) and Dibai et al. explicitly drew attention to it. We cannot say whether the differences in the profiles obtained by ourselves and Dibai $e$ t al. and by Anderson are real. No convincing systematic changes between December 1969 and June 1970 were noted. Dibai et al. also called attention to some apparent finer structure in the profiles; there is a suspicion of this in our material, but it is by no means confirmed.

The main conclusion to be drawn from this asymmetry is simply that electron scattering cannot be the sole principle broadening agent; mass motions of at least $2500 \mathrm{~km} \mathrm{~s}^{-1}$ are involved. Dibai et al. proposed that radial expansion was involved, and, assuming an electron density in this region of $10^{7} \mathrm{~cm}^{-3}$ deduced a characteristic 
time of $30 \mathrm{yr}$. The density could very well be as high as $10^{9} \mathrm{~cm}^{-3}$ however, in which case the time would be only $1 \mathrm{yr}$. If the gas is bound by some massive object it is hard to see how the motion could persist, unless it is fairly well ordered, which suggests rotation. The asymmetry could then be due either to obscuring material or to clumpi-
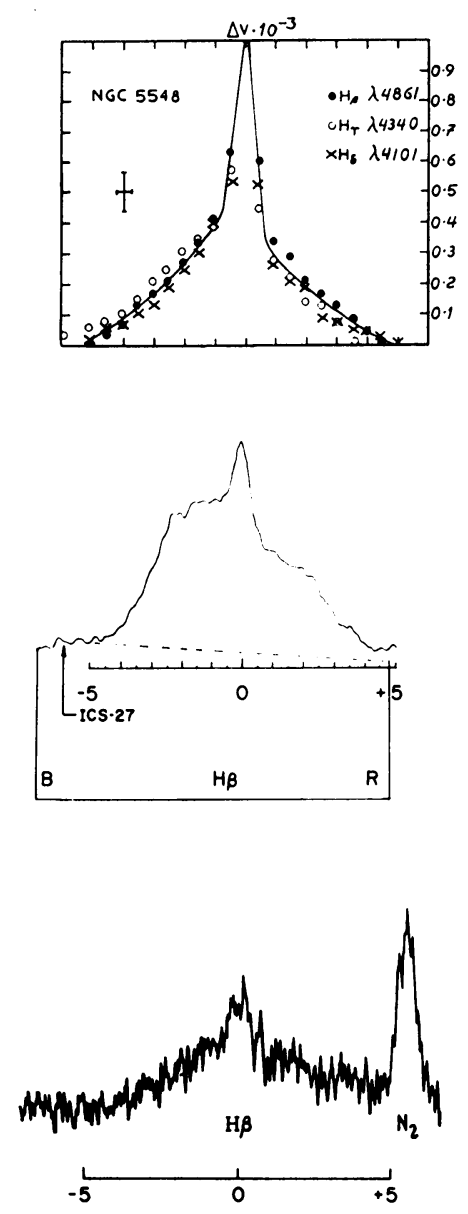

Fig. 1. Profiles of $\mathrm{H} \beta$ in the Seyfert galaxy NGC 5548. The top profile is based on work of Anderson (1970), the middle profile is an intensity tracing of a spectrogram taken by the present authors in January 1970, and the bottom profile is from a spectrogram of Dibai et al. taken in May 1966.

ness. In either case, one anticipates changes in the spectrum on a time scale of perhaps a few months to $30 \mathrm{yr}$.

Anderson and Kraft (1969) have discussed the displaced absorption cores of HeI 3889 and the Balmer lines in NGC 4151 and derived from these features very high mass ejection rates. Subsequent observation by us (Cromwell and Weymann, 1970) in December 1969 and January 1970 showed that the $\mathrm{H} \beta$ absorption was quite strong, 
and must be transient. This fact, together with the presumption that these features were formed closer to the nucleus than was assumed by Anderson and Kraft, led us to an estimate of the mean mass ejection rate very much lower than Anderson and Kraft's.

By late April, this absorption core had weakened to the point where, with our resolution, it had disappeared. Figure 2 shows microphotometer tracings of plates

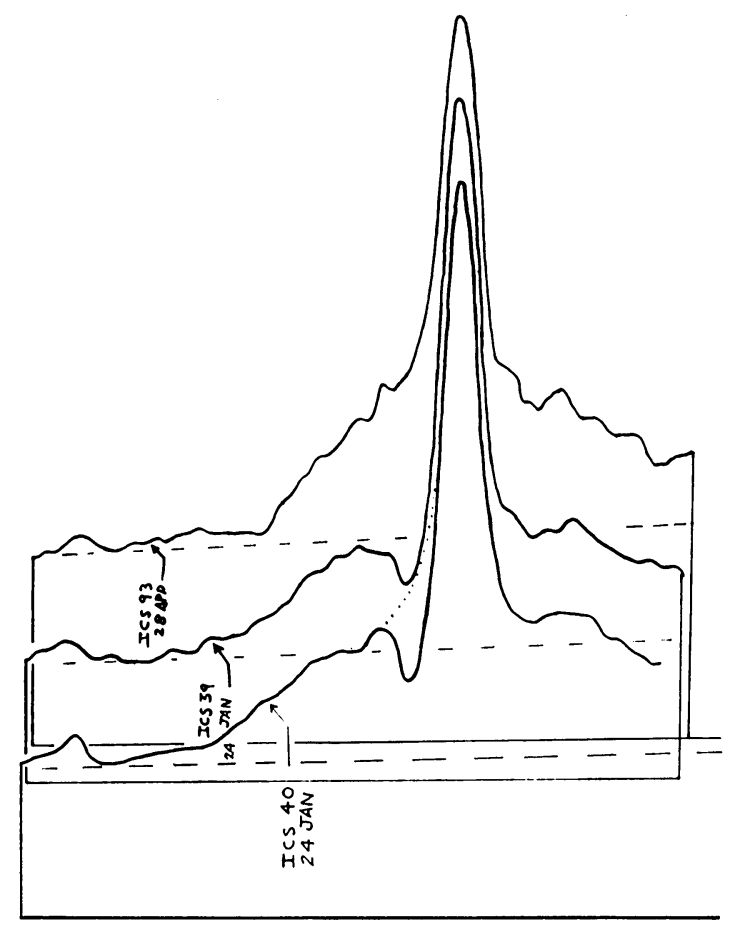

Fig. 2. Profiles of $\mathrm{H} \beta$ in the Seyfert galaxy NGC 4151. The bottom two profiles were taken on the same night (January 24) and show the displaced absorption core. The top profile was taken April 28, by which time, at our resolution, the absorption core had disappeared.

taken in January, and one in April. This displacement of the core from the line center is $1000 \mathrm{~km} \mathrm{~s}^{-1}$ so that during these three months the material would have moved only $10^{15} \mathrm{~cm}$.

The interpretation is complicated by the fact that the nucleus was abnormally faint during December and January. (Babadzhanjanz et al., 1970). This is also evident from the spectrograms at this epoch, which show a large number of faint emission lines normally undetectable. We consider now the following possibilities for the rapid change, in order to see whether this very small dimension has any physical significance.

(a) The feature is permanently present, but is normally masked by the non-thermal continuum;

(b) a distant small cloud passed in front of the nucleus; 
(c) the optical depth in $\mathrm{H} \beta$ of an intervening cloud changed due to changes in excitation and ionization associated with the fluctuation in brightness of the nucleus;

(d) a shell of gas was expelled from the nucleus.

We cannot exclude (a) with certainty, but feel it is not likely for the following reasons: The absorbing material would have to be seen projected against only the broad emission wings, rather than the continuum, requiring the continuum and broad emission to arise from quite different regions of space. Moreover, the limited material at our disposal suggests that the ratio of the continuum intensity to the broad emission wing intensity remained roughly constant during December-April, so that 'filling in' would not have occurred anyway. Possibility (b) is highly unlikely: the cloud would have to be of the order $10^{15} \mathrm{~cm}$ and very dense, but ionized, to produce the required optical depth. In order not to have the passage of such a cloud across the nucleus be exceedingly unlikely, there must be enormous numbers of such clouds, in which case the emission from them would exceed by many orders of magnitude that actually observed.

Possibilities (c) and (d) have been considered together by solving the ionization equilibrium and statistical equilibrium equations governing the population of the $2 s$ and $2 p$ states in hydrogen and the $2 s^{3} S$ state of neutral helium. We assume photoionization from a point source with a power law extrapolation of the visible radiation into the far UV. The details will be published elsewhere, but the main conclusions are as follows:

(1) Of the four processes considered for controlling the rate at which electrons leave the $2 s$ and $2 p$ states (namely: two photon decay from $2 s ; 2 s-2 p$ collisions followed immediately by Ly- $\alpha$ escape ; $2 s-s p$ equilibrium with occasional $\mathrm{L} \gamma-\alpha$ escape; photoionization from the $n=2$ level) we find that only the third leads to acceptable time scales, and suggests a thin shell of radius $R=10^{16} \mathrm{~cm}$, density $10^{10} \mathrm{~cm}^{-3}$ during the outburst. For a thin, expanding shell it can be shown in this case that the optical depth in the Balmer lines varies roughly as $R^{-8}$.

(2) An increase in the optical depth of $\mathrm{H} \beta$ in a shell due to a decrease in the intensity of the ionizing flux over a three month period is plausible only in the Lyman- $\alpha$ escape regime described above. In particular, for lower values of $N_{e}$ and slightly larger values of $R$ where the rate of escape of electrons from the $2 s$ state is governed by the rate of $2 s-s p$ collisions, increasing the ionizing flux does not affect the optical depth of $\mathrm{H} \beta$.

(3) A slab with $N_{e} \sim 10^{10} \mathrm{~cm}^{-3}, R \sim 10^{16} \mathrm{~cm}$ and thickness such that $\tau(\mathrm{H} \beta) \sim 1$ would have $\tau(3889) \ll 1$.

(4) Material which accumulates in a fairly narrow strip characterized by $N_{e} \sim 10^{7}$, $R \sim 3 \times 10^{17} \mathrm{~cm}$ down to $N_{e} \sim 10^{4}, R \sim 10^{19} \mathrm{~cm}$ can have $\tau(3889) \sim 1$ with $\tau(\mathrm{H} \beta) \sim \frac{1}{10}$.

A model for this spectroscopic behavior which seems not grossly to contradict the observed facts thus involves the frequent expulsion of shells or filaments, each of which produces a short lived displaced $\mathrm{H} \beta$ core, but whose cumulative effect is to produce a relatively more stable He 3889 displaced core. The mass loss rate associated with this picture is probably intermediate between that suggested by Anderson and Kraft and by Cromwell and Weymann. 


\section{References}

Anderson, K.: 1970, Astrophys. J. 162, 743.

Anderson, K. and Kraft, R. P.: 1969, Astrophys. J. 158, 859.

Babadzhanjanz, M. K., Hagen-Thorn, V. A., and Ljutyi, V. M.: 1970, Astron. Cirk. Izdav. Bjuro Astron. Soobshch. Acad. Sci. U.S.S.R., No. 544.

Cromwell, R. and Weymann, R.: 1970, Astrophys. J. Letters 159, L147.

Dibai, E. A., Yesipov, V. F., and Pronik, V. I.: 1968, Soviet Astron. 11, 553.

Mathis, J.: 1970, Astrophys. J. 162, 761.

Weymann, R.: 1970, Astrophys. J. 160, 31. 